\title{
A morphometric technique for analysis of cochlear vessels
}

\author{
Lynn Carlisle Shaddock ${ }^{1}$, Roger P. Hamernik ${ }^{2}$ and Charles G. Wright ${ }^{3}$ \\ ' Kresge Hearing Research Institute, University of Michigan, I301 E. Ann St., Ann Arbor, MI 48109. \\ Callier Center for Communication Disorders, University of Texas at Dallas, 1966 Inwood Road, Dallas, TX 75235. and \\ ${ }^{3}$ Department of Otolaryngology. University of Texas Health Science Center at Dallas. 5323 Harry Hines Blvd., Dallas. TX 75235. U.S.A.
}

(Received 19 June 1985; accepted 20 August 1985)

\begin{abstract}
A computer based system was developed for morphometric analysis of the cochlear lateral wall vasculature. Variables measured were vascular density, RBC density, vessel width, aggregation density, lumen compression count, WBC count and pigment density. Vessels were divided into three systems based on the lateral wall structures that they supplied. The results indicated that these three vessel systems were morphologically distinct in terms of their vascular density, RBC density, and vessel width. How these differences relate to functional needs of the lateral wall is discussed.
\end{abstract}

morphometrics, cochlear microvasculature, stria vascularis. spiral ligament. spiral prominence

\section{Introduction}

Changes in the vascular system of the cochlea have been suggested as a cause of many forms of hearing impairment; among these are Ménière's disease, sudden deafness, tinnitus and noise-induced hearing loss (Lawrence, 1983). It has been difficult to investigate microvascular involvement in such conditions through experimentation because the vasculature of the cochlea does not readily lend itself to quantitative evaluation.

Many studies have been published describing changes in cochlear vessels after exposure to ototraumatic agents such as noise and ototoxic drugs. Over the past 10 years, Axelsson and colleagues (Axelsson et al., 1974, 1975; Vertes and Axelsson, 1979) have developed a method based on a subjective evaluation of 20 vascular parameters that are likely to be affected by cochlear trauma. Their 'soft-surface specimen technique' permits evaluation of all the vessels over the entire length of the cochlea so that a comprehensive picture of the vasculature, both in terms of the involvement of the various vessel groups and the location of damage, can be drawn from the results of the analysis. Santi and colleagues (Santi and Lakhaani, 1983; Santi et al., 1983) have used a stereological technique to measure relative areas of the three cell layers and the capillaries in the stria vascularis. $\Lambda$ related technique, computerized image analysis, was recently used by Smith et al. (1985) to measure capillary density and erythrocyte distribution in strial capillaries.

The objective of this study was to develop a computer-based technique to quantify a number of vascular variables in all of the lateral wall vessels. Early studies of the cochlea describe specific vascular pathways through the lateral wall (Axelsson, 1968; Smith, 1951); some vessels pass through the spiral ligament from scala vestibuli to scala tympani, while others form the capillary beds of the stria vascularis or the spiral prominence. However, most studies to date have either disregarded all vessels other than those in the stria vascularis (Santi and Lakhani, 1983; Santi et al., 1983; Smith et al., 1985) or have divided the vasculature into groups based on location in scala vestibuli, scala media or scala tympani (Axelsson, 1968; Axelsson et al., 1974, 1975; Vertes and Axelsson, 1979). In this study, the lateral wall vessels were divided into three systems based on the structure (spiral ligament, stria vascularis, or spiral prominence) that they supply. If these structures react differently to trauma, such differences should be reflected in the 
vessels. By analyzing the vessels by 'system', we may gain a clearer picture of the normal function of the microvessels and of how they change in response to cochlear trauma.

\section{Method}

Five healthy adult chinchillas, all of the same coat color and all less than eight years of age, were used in the study. Three animals were female and two were male. The animals were anesthetized by subcutaneous injection of $0.15 \mathrm{ml}$ Ketamine (100 $\mathrm{mg} / \mathrm{ml})$ with acepromazine $(10 \mathrm{mg} / \mathrm{ml})$ and xylazine $(20 \mathrm{mg} / \mathrm{ml})$ in a $3: 1: 3$ mixture. The animals were decapitated, the temporal bones removed and the cochleas perfused through the round and oval windows with $2.5 \%$ glutaraldehyde in veronal acetate buffer ( $\mathrm{pH} 7.4)$; then the temporal bones were immersed in fixative and stored overnight under refrigeration. The following day each cochlea was very lightly post-fixed with $1 \%$ osmium tetroxide by perfusing the solution through the cochlea and then immediately flushing the perilymphatic space with $50 \%$ ethanol. Following dehydration to $70 \%$ ethanol, a piece of spiral ligament with the stria vascularis attached was dissected from the cochlea, rehydrated and then bleached in $3 \%$ hydrogen peroxide for $5 \mathrm{~min}$ to remove excess osmium so that the vessels could be more easily visualized. Each dissected specimen was approximately $3 \mathrm{~mm}$ in length and came from a location which represented $50 \%$ of the distance from the apical end of the spiral ligament. The tissue was mounted in glycerol with the stria vascularis facing upward on glass slides.

The computer system used to collect and analyze the data is illustrated schematically in Fig. 1. A low-light, high-resolution black-and-white video camera (RCA 2000 Ultracon) was attached to the photo tube of a Zeiss Universal compound microscope. The camera signal, along with the signal from the graphics unit (Summagraphics 2000) and the CPU (DEC LSI 11-23) were synchronized so that the microscopic image, the image drawn on the graphics tablet and messages generated by the software were simultaneously displayed on the video-monitor screen. To measure an object displayed on the monitor screen, the object was traced on the graphics tablet using a four-button
IMAGE ANALYSIS SYSTEM

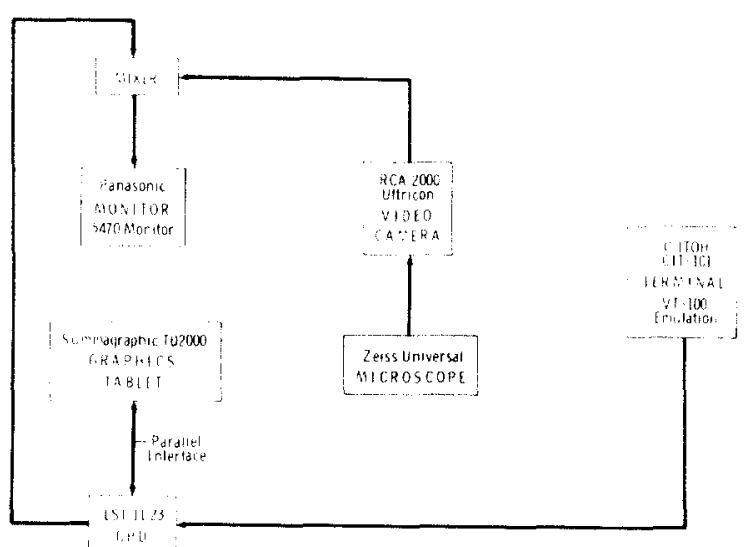

Fig. 1. Schematic diagram of the image analysis system used to collect and analyze the vascular data. The microscopic image is projected via the video camera to the monitor screen. The graphics tablet and CPU are also patched into the monitor so that objects on the monitor screen can be traced on the data tablet.

cursor. Each button or combination of buttons controlled a different function of the software. As the object was traced, a series of $(X, Y)$ pairs which corresponded to the outline of the object was stored in files in the CPU for later analysis.

This image analysis system was used to evaluate the vessels of the lateral wall. Every vessel was assigned to one of three systems. The first system consisted of the vessels feeding into the stria vascularis, the vessels of the stria vascularis and the vessels draining the stria vascularis. The second system consisted of the vessels of the spiral ligament. The third system consisted of the vessels feeding into the spiral prominence, the vessel of the spiral prominence and the vessels draining the spiral prominence. In some cases, there was a branching of vessels in scala vestibuli and the two branches went to different systems. When this occurred, the part of the vessel above the bifurcation point was considered to be part of the same vessel as the larger of the two branch vessets. The three vessel systems are illustrated schematically in Fig. 2.

Measurements were made over an area of approximately $1 \mathrm{~mm}^{2}$ which was divided into 12 adjacent fields, as illustrated in Fig. 3. Within each field, and for each vessel, the following measure- 

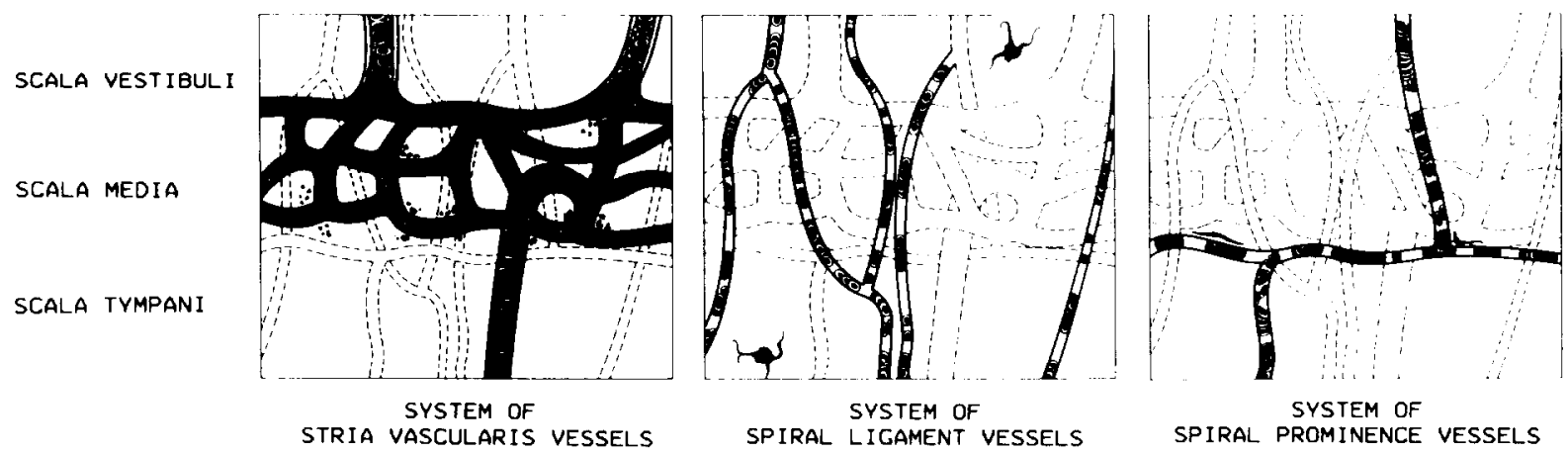

Fig. 2. Artist's conception of the three vessel systems of the lateral wall. Each panel highlights one system and the other two are represented by dotted lines.

ments were made:

1. The area of field was quantified by tracing the outline of the tissue.

2. The area of the vessels which belonged to each system was quantified by tracing the outlines of the appropriate vessels.

3. The area occupied by red blood cells within the vessels of each system was quantified by tracing the outlines of RBCs contained within the appropriate vessels.

4. The total length of the vessels of each system was quantified by tracing down the center of the appropriate vessels.

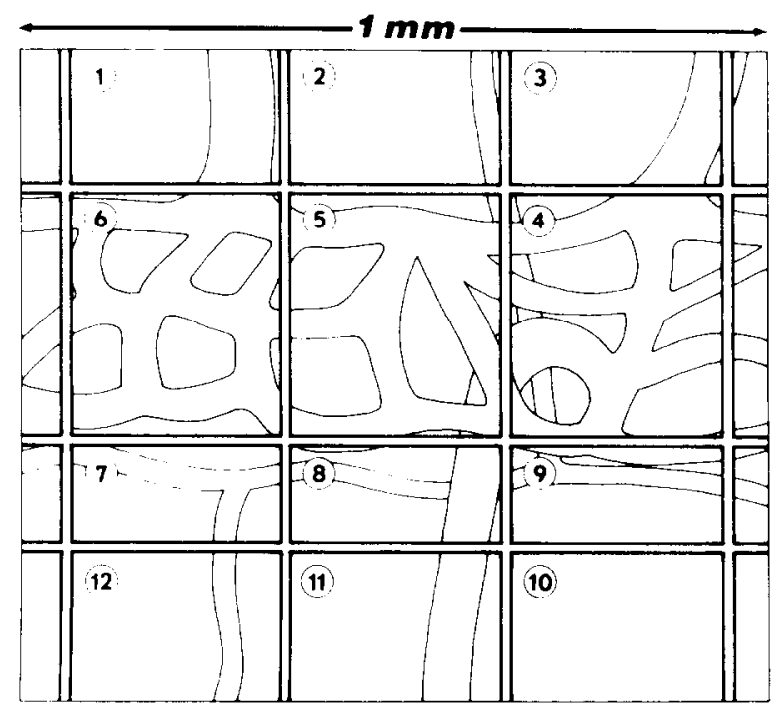

Fig. 3. Schematic representation of the 12 microscopic fields analyzed in each specimen. Fields $1-3$ are located in scala vestibuli, fields 4-9 are located in scala media, and fields $10-12$ are located in scala tympani.
5. The area occupied by aggregations of RBCs in the vessels of each system was quantified by tracing the outlines of abnormal aggregations of RBCs in the appropriate vessels.

6. The number of white blood cells in the vessels of each system was counted.

7. The number of lumen compressions in the vessels of each system was counted.

8. The area occupied by pigment cells and pigment granules was quantified by tracing the outlines of pigment cells and granules in the field.

Since the field area was constant for all three vessel systems, that measurement was taken only once per field. All other measurements were made three times; once for each vessel system. Therefore, a total of 22 measurements were made for each field ( 7 measurements $\times 3$ systems + area of field). When all of the measurements for a single specimen were completed, a preliminary analysis program computed the areas and tabulated the counts for all 264 measurements ( 22 measurements $\times 12$ fields). These values were then used to computer seven variables, which were:

1. Vascular density: $\frac{\text { area occupied by vessels }}{\text { area of field }}$

2. RBC density: $\frac{\text { area occupied by RBCs }}{\text { area occupied by vessels }}$

3. Vessel width: $\frac{\text { area occupied by vessels }}{\text { length of vessels }}$

4. Aggregation density: area occupied by aggregations of RBCs area occupied by RBCs 
5. Relative frequency of WBCs: $\frac{\text { number of WBCs } \times 1000^{*}}{\text { area occupied by vessels }}$

6. Relative frequency of lumen compression: number of compressions $\times 1000 *$ area occupied by vessels

7. Pigment density: $\frac{\text { area occupied by pigment }}{\text { area of field }}$

For the statistical analysis, the data were combined to reflect the location of the vessels with respect to the three cochlear scalae; fields 1-3 were located adjacent to scala vestibuli, fields 4-9 were located adjacent to scala media and fields 10-12 were located adjacent to scala tympani. The data collected in scala media was further subdivided into two areas; fields 4-6 represented the stria vascularis and the area of spiral ligament behind the stria vascularis and fields 7-9 represented the spiral prominence and the area of spiral ligament behind the spiral prominence.

One-way analysis of variance tests were used to evaluate the data. For each of the seven vascular variables, the three vessel systems were compared. The test was performed four times; for data col-

* Since these numbers were expected to be small compared to the denominators, counts were multiplied by 1000 . lected in scala vestibuli (fields $1-3$ ), in the stria vascularis portion of scala media (fields 4-6), in the spiral prominence portion of scala media (fields 79 ) and in scala tympani (fields 10.12 ).

\section{Results}

Two of the dependent variables that had been evaluated, WBC count and lumen compression count, were eliminated from the statistical analysis because no examples of either variable had been found during data collection. The means and S.D. of the five remaining variables are contained in Table I.

Since pigment cells and pigment granules are contained in the tissue rather than in the vessels, in scala vestibuli (fields $1-3$ ) and scala tympani (fields 10-12) pigment density could only be measured in the spiral ligament. In fields $4-6$ of scala media, pigment density was measured in the stria vascularis and in the spiral ligament behind the stria, and in fields 7-9 pigment density was measured in the spiral prominence and in the spiral ligament behind the spiral prominence. No pigmentation was observed in the tissue of the spiral ligament in scala media behind the stria or spiral prominence; all pigment cells and granules seen in

TABLE I

MEANS AND S.D. BY VESSEL SYSTEM AND LOCATION

\begin{tabular}{|c|c|c|c|c|c|c|c|c|c|c|}
\hline & \multicolumn{2}{|c|}{ Vascular density } & \multicolumn{2}{|c|}{ RBC density } & \multicolumn{2}{|c|}{ Vessel width } & \multicolumn{2}{|c|}{ Aggregation density } & \multicolumn{2}{|c|}{ Pigment density } \\
\hline & $\bar{x}$ & S.D. & $x$ & S.D. & $\bar{x}$ & S.D. & $\bar{x}$ & S.D. & $x$ & S.D. \\
\hline \multicolumn{11}{|c|}{ System of stria vascularis vessels } \\
\hline Scala vestibuli & 0.0516 & 0.0278 & 0.5406 & 0.2465 & 14.238 & 2.454 & 0.0000 & 0.0000 & - & - \\
\hline Scala media (stria) & 0.1868 & 0.0487 & 0.8711 & 0.2679 & 7.447 & 1.502 & 0.0000 & 0.0000 & 0.0062 & 0.0068 \\
\hline Scala media (prominence) & 0.0391 & 0.0198 & 0.6877 & 0.0665 & 11.829 & 2.271 & 0.0000 & 0.0000 & - & $\ldots$ \\
\hline Scala tympani & 0.0299 & 0.0140 & 0.6025 & 0.2619 & 11.117 & 2.520 & 0.0000 & 0.0000 & - & - \\
\hline \multicolumn{11}{|c|}{ System of spiral ligament vessels } \\
\hline Scala vestibuli & 0.0622 & 0.0217 & 0.3002 & 0.1893 & 6.822 & 1.168 & 0.0269 & 0.1041 & 0.0045 & 0.0056 \\
\hline Scala media (stria) & 0.0588 & 0.0159 & 0.2902 & 0.0908 & 5.485 & 1.053 & 0.0000 & 0.0000 & 0.0000 & 0.0000 \\
\hline Scala media (prominence) & 0.0472 & 0.0169 & 0.4697 & 0.1514 & 5.846 & 1.162 & 0.0000 & 0.0000 & 0.0000 & 0.0000 \\
\hline Scala tympani & 0.0553 & 0.0186 & 0.4533 & 0.1576 & 5.895 & 1.586 & 0.0000 & 0.0000 & 0.0067 & 0.0100 \\
\hline \multicolumn{11}{|c|}{ System of spiral prominence vessels } \\
\hline Scala vestibuli & 0.0276 & 0.0165 & 0.5261 & 0.1588 & 10.102 & 2.316 & 0.0000 & 0.0000 & - & - \\
\hline Scala media (stria) & 0.0220 & 0.0165 & 0.4866 & 0.0923 & 7.088 & 1.016 & 0.0000 & 0.0000 & - & - \\
\hline Scala media (prominence) & 0.0713 & 0.0265 & 0.6520 & 0.1196 & 6.733 & 1.409 & 0.0259 & 0.1003 & 0.0030 & 0.0039 \\
\hline Scala tympani & 0.0282 & 0.0076 & 0.4215 & 0.1124 & 6.578 & 1.723 & 0.0000 & 0.0000 & - & - \\
\hline
\end{tabular}


TABLE II

RESULTS OF ONE-WAY ANOVA

\begin{tabular}{|c|c|c|c|c|c|c|c|c|}
\hline & \multicolumn{2}{|c|}{ Vascular density } & \multicolumn{2}{|c|}{ RBC density } & \multicolumn{2}{|c|}{ Vessel width } & \multicolumn{2}{|l|}{ Aggregation } \\
\hline & $\bar{F}$ & $P$ & $\bar{F}$ & $P$ & $\bar{F}$ & $P$ & $\bar{F}$ & $P$ \\
\hline $\begin{array}{l}\text { Scala vestibuli } \\
\left(F_{2.29}\right)\end{array}$ & 6.5950 & $<0.0044$ & 5.4242 & $<0.0100$ & 43.0050 & $<0.0000$ & 0.5502 & 0.5827 \\
\hline $\begin{array}{l}\text { Scala media (stria) } \\
\qquad\left(F_{2,36}\right)\end{array}$ & 94.3858 & $<0.0000$ & 39.2597 & $<0.0000$ & 10.3068 & $<0.0003$ & not performed & \\
\hline $\begin{array}{l}\text { Scala media (prominence) } \\
\quad\left(F_{2,32}\right)\end{array}$ & 6.9499 & $<0.0031$ & 9.4446 & $<0.0006$ & 32.7421 & $<0.0000$ & 0.6531 & $<0.5272$ \\
\hline $\begin{array}{l}\text { Scala tympani } \\
\quad\left(F_{2.25}\right)\end{array}$ & 11.3997 & $<0.0003$ & 1.8086 & $<0.1847$ & 13.9656 & $<0.0001$ & not performed & \\
\hline
\end{tabular}

scala media were in the tissue of the stria vascularis and the spiral prominence.

Aggregation density was only measured in two cases; for the vessels of the spiral ligament in scala vestibuli and in the vessel of the spiral prominence.

The results of the one-way ANOVA tests for vascular density, $\mathrm{RBC}$ density, vessel width and aggregation density are given in Table II. The three vessel systems were significantly different ( $P<0.05$ or less) for vascular density and vessel width at all locations and for RBC density at all locations except scala tympani. There was no significant difference between the vessel systems for aggregation density. ANOVA could not be per-

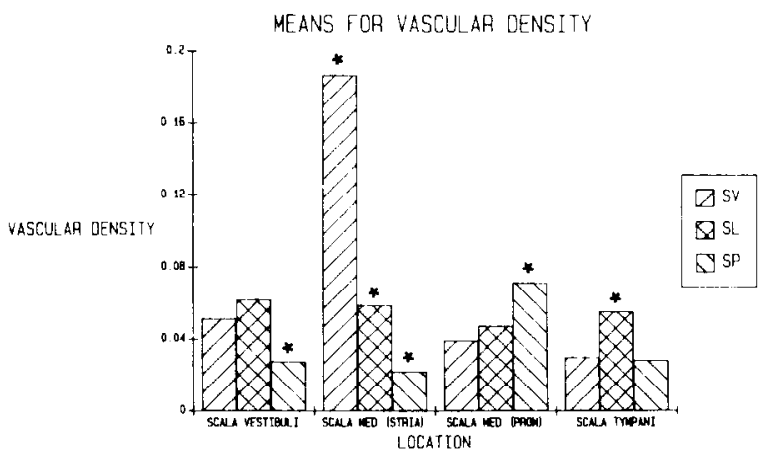

Fig. 4. Mean values for the variable vascular density obtained for each scala location for all three vessel systems. Asterisks indicate a statistically significant difference as revealed by the SNK test. SV, system of stria vascularis vessels; SL, system of spiral ligament vessels, SP, system of spiral prominence vessels. formed on pigment density since all systems were not represented at any location.

For all cases where the ANOVA revealed a significant difference between the three systems, the Student-Newman-Keuls (SNK) multiple range test was used to determine which system or systems accounted for the difference (Zar, 1980).

Fig. 4 is a graph of the mean values obtained for vascular density for each vessel system at each location. Asterisks indicate which system(s) mean was significantly different from the others as revealed by the SNK procedure. In scala vestibuli, mean vascular density was significantly less in the system of spiral prominence vessels than in the other two vessel systems. In the stria vascularis

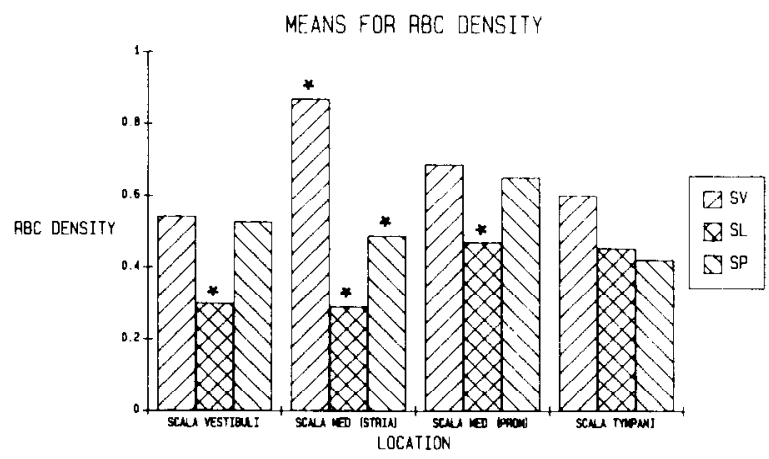

rig. 5. Mean values for the variable RBC density obtained for each scala location for all three vessel systems. Asterisks indicate a statistically significant difference as revealed by the SNK test. SV, system of stria vascularis vessels; SL, system of spiral ligament vessels; SP, system of spiral prominence vessels. 


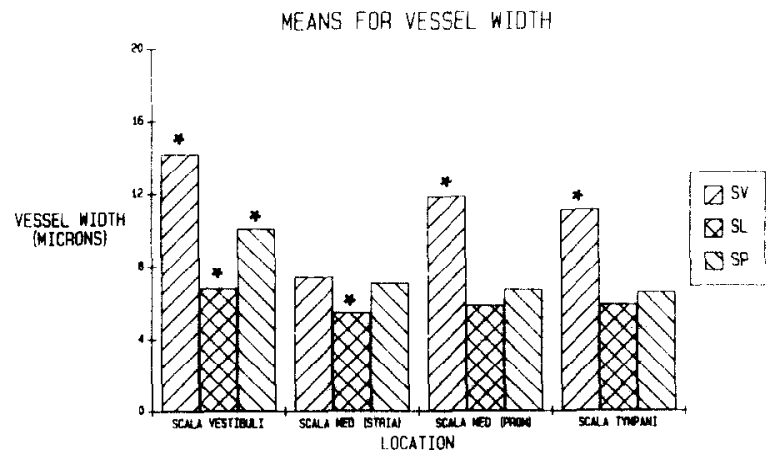

Fig. 6. Mean values for the variable vessel width obtained for each scala location for all three vessel systems. Asterisks indicate a statistically significant difference as revealed by the SNK test. SV, system of stria vascularis vessels; SL, system of spiral ligament vessels; SP, system of spiral prominence vessels.

portion of scala media, each vessel system had a mean value for vascular density that was significantly different from the other two systems. Vascular density was over three times greater in the system of stria vascularis vessels than in the system of spiral ligament vessels, and over eight times greater than in the system of spiral prominence vessels.

In the spiral prominence portion of scala media, the mean value for vascular density was significantly greater in the system of spiral prominence vessels than in the other two vessel systems. Vascular density was significantly greater in the system of spiral ligament vessels than in the other two vessel systems in the scala tympani.

Mean values for RBC density are displayed in Fig. 5. RBC density was significantly lower in the system of spiral ligament vessels than in the other two systems in scala vestibuli and in scala media. In the stria vascularis portion of scala media, RBC density was three times greater in the system of stria vascularis vessels than in the vessels of the spiral ligament and almost two times greater than in the system of spiral prominence vessels.

Fig. 6 shows the mean values for vessel width in each vessel system and location. Mean vessel width was significantly greater for the system of stria vascularis vessels than the other two vessel systems at all locations other than the stria vascularis portion of scala media. Vessel width was significantly less for the vessels of the spiral ligament than for the other two vessel systems in scala vestibuli and in the stria vascularis portion of scala media.

\section{Discussion}

The purpose of this study was to develop a quantitative method for evaluating the microvasculature of the cochlea and to use that method to determine if the vessel systems of the stria vascularis, spiral ligament and spiral prominence were morphologically distinct. This method expands the image-analysis technique used by Smith et al. (1985) to include all the vessels of the lateral wall, permitting more vascular variables to be analyzed as in the 'soft-surface technique' of Vertes and Axelsson (1979). The results indicate that the three vessel systems are morphologically distinct in terms of vascular density, $R B C$ density and vessel width. Since only $1 \mathrm{~mm}^{2}$ was analyzed, we cannot evaluate the basal or apical shunting of blood.

An important function of the stria vascularis is to maintain the high potassium level of endolymph (Bosher and Warren, 1968) and the high positive endolymphatic potential that is necessary for energy transduction in the organ of Corti (Tasaki and Spyropoulos, 1959). The average width of the vessels feeding the stria is greater than the average width of the vessels draining the stria, and the vascular density is higher in scala vestibuli than in scala tympani, which means that there are more vessels feeding stria vascularis than there are draining stria vascularis. This creates a 'bottleneck' (Fulton, 1970) for blood draining out of the stria, which in turn contributes to the very high $\mathrm{RBC}$ density in the vessels of the stria vascularis and helps to explain why the RBC density is higher in the vessels draining the stria than in those feeding the stria; there are fewer channels flowing out than flowing in.

The functional importance of the spiral prominence is less well documented than the stria vascularis, but there are structural features that suggest that the spiral prominence is involved in fluid transport. Many similarities exist between the stria vascularis and the spiral prominence. Both structures contain an epithelial cell layer with tight cell junctions which prevent diffusion into or out of the endolymph (Forge, 1981; Spoendlin, 1967). In the stria vascularis, the epithelial cells are the 
marginal cells; in the spiral prominence the epithelium consists of a single layer of small cuboidal or flat cells. The stroma cells of the spiral prominence show a high content of mitochondria, endoplasmic reticulum and Golgi membranes which reflect the high metabolic activity of these cells (Vosteen. 1960) similar to the marginal cells of the stria vascularis. Both the spiral prominence and the stria vascularis are highly vascularized, but the vessels of the stria vascularis are closely surrounded by the marginal cell processes while the vessels of the spiral prominence run free in the extracellular space, in the area occupied by stroma cells and the roots of the outer sulcus cells (Spoendlin, 1967). The extensive plasma membrane infoldings and the large extracellular space, along with evidence for a resorptive function of the outer sulcus cells (Spoendlin, 1967) all indicate that the spiral prominence is involved in fluid transport. Although the numbers are different, the patterns for vascular density, RBC density and vessel width in the system of spiral prominence vessels resemble those seen in the system of strial vessels. In both systems, vascular density and $\mathrm{RBC}$ density increase substantially in the 'target' tissue, i.e., the stria vascularis and the spiral prominence, and then decrease in the draining vessels in scala tympani. In both systems, the diameter of the draining vessels is substantially less than that of the feeding vessels, ensuring that blood flow through the 'target' tissue will be slow enough to maximize nutrient exchange.

Unlike the system of stria vascularis vessels and the system of spiral prominence vessels, there is no specific, metabolically active area which is the 'target' of the vessels of the spiral ligament (Smith. 1973; Hawkins, 1973), although Hawkins has described the area of spiral ligament above Reissner's membrane as one of the loci of perilymph formation. It is not surprising, then, that the morphology of the vessels in this system does not change much as they pass from scala vestibuli through scala media to scala tympani, although they do taper and RBC density increases accordingly.

A surprising finding in this study was the complete absence of perivascular cells and endothelial cells compressing the vessel lumen. Such compressions are seen in normal cochleas (Smith et al.,
1985) and their absence in this tissue may have been related to the anesthesia, since anesthetics are known to cause alterations in microvascular function (Longnecker, 1980).

Ketamine has been shown to cause dilation of arterioles (Longnecker et al., 1974), perhaps via changes in the sympathetic tone of the vascular system. If a similar mechanism exists in the cochlear vessels, then Ketamine would be expected to lower cochlear blood pressure by dilating the spiral modiolar artery. It has been demonstrated (Mellander and Johansson, 1968) that decreases in hydrostatic pressure cause dilation of precapillaries and arterioles. This dilation could result in an increase in blood flow to the lateral wall capillaries and a concomitant increase in intraluminal pressure that would account for the absence of lumen compressions. Another possible explanation is that the anesthetic acted directly on the pericytes of the capillaries, although the contractility of pericytes remains a controversial topic. There is no direct evidence to support the idea of contractility of pericytes in mammals (Zweifach, 1971; Folkow and Neil, 1971); however, these cells have been shown to contain actin filaments (Kimura, 1962) and actomyosin (Majno, 1969). If pericytes contain contractile elements then perhaps the mechanisms by which anesthetics relax smooth muscle cells around larger vessels also causes relaxation of the contractile elements in pericytes.

Although pigment density could not be analyzed statistically because of its localization to a few specific areas within certain structures of the lateral wall, pigment density may be an important variable to evaluate when pathologic tissue is involved. It has been shown (Hilding and Ginzberg. 1977) that all intermediate cells in the rat stria vascularis exhibit all stages of melanogenesis and are by definition melanocytes. Melanin granules have been described in the marginal cells as well (Rodriguez-Eschandia and Burgos, 1965) and it has been suggested that melanin granules are "donated" to the marginal cells by intermediate cells (Hilding and Ginzberg, 1977) much as the melanocytes in the epidermis transfer melanin granules to the malphigian cells (Fitzpatrick et al., 1961). While the pigmentation visible at the light microscopic level in normal stria vascularis con- 
sists of scattered golden brown pigment cells and pigment granules, on the spiral prominence melanocytes are spindle shaped and lie along the vessel of the spiral prominence. On the spiral ligament in scala vestibuli and scala tympani, large, dense dendritic-type melanocytes are found, usually in conjunction with a blood vessel. The role of melanin pigmentation in the inner ear is unclear, although it has been implicated as a contributing factor in aminoglycoside ototoxicity (Comis and Leng, 1980; Lyttkins et al., 1979) and noise trauma (Conlee et al., 1985).

The technique described in this paper for the analysis of cochlear vessels has allowed us to collect statistical data concerning several features of normal vessel morphology. The significance of these results is limited by the small sample size as well as by the restricted area of the lateral wall over which measurements were made. Our principle aim in this study was to determine if the three vessel systems, as we have defined them, were in fact morphologically distinct from each other. Our results indicate that they are within our very restricted sample area. Obviously, the distinction needs to be demonstrated for other areas of the cochlea and for other experimental animals. Since several of the vascular variables we have used may be influenced by method of sacrifice (Santi and Duvall, 1979), our measurements are intended to be relative rather than absolute. Method of sacrifice poses significant problems for histological studies of the vasculature. Anesthesia affects the microcirculation by causing dilation of arterioles and sometimes venules (Longnecker, 1980), and such effects could be reflected in measurements of vessel size. The alternative method is decapitation without anesthesia, which could result in a different set of artefactual changes related to a stress response. The extent to which method of sacrifice affects the microvasculature of the inner ear has not been studicd systematically. A morphometric technique such as the one described in this paper could be used to investigate this issue.

\section{Acknowledgements}

The authors wish to thank Dr. William Ahroon for writing the software used in this project, and Mr. George Turrentine for technical assistance.
This project was supported by National Institute of Occupational Safety and Health grant No. 1 RO1 OH1518-01 and U.S. Army Medical Research and Development Command grant No. DAMD 17-83-G-9555.

\section{References}

Axelsson, A. (1968): The vascular anatomy of the cochlea in the guinea pig and in man. Acta Otolaryngol. Suppl. 243.

Axelsson, A., Miller, J. and Holmquist, J. (1974): Studies of cochlear vasculature and sensory structures: A modified method. Ann. Otol. 83, 537-549.

Axelsson, A., Miller, J. and Larsson, B. (1975): A modified 'soft surface specimen technique' for examination of the inner ear. Acta Otolaryngol. 80, 362-375.

Bosher, S.K. and Warren, R.L. (1968): Observations on the electrochemistry of the cochlear endolymph in the rat: A quantitative study of its electrical potential and ionic composition as determined by means of flame spectrophotometry. Proc. R. Soc. London Ser. B 171, 227-247.

Comis, S.D. and Leng, G. (1980): Kanamycin ototoxicity and pigmentation in the guinea pig. Hearing Res. 3, 249-251.

Conlee, J.W., Abdul-Baqui, K.J.Y., Creel, D.J., Parks, T.N. and McCandless, G.A. (1985). Differential susceptibility to noise-induced permanent threshold shift in albino and pigmented guinea pigs. Abstract presented at Eighth Midwinter Research Meeting, Association for Research in Otolaryngology, p. 85.

Fitzpatrick, T.B., Seiji, M. and McGugan, A.D. (1961): Melanin pigmentation. N. Engl. J. Med. 328-331.

Folkow, B. and Neil, E., eds. (1971): The Viscosity Factor. In: Circulation, p. 29. Oxford University Press, Oxford.

Forge, A. (1981): Electron microscopy of the stria vascularis and its response to ethacrynic acid. Audiology 20, 273-289.

Fulton, G.P. (1970): Structure and function of the microvascular system. In: Microcirculation, Perfusion and Transplantation of Organs, pp. 9-21. Editors: T.I. Malinin, B.S. Linn, A.B. Callahan and W.D. Warren. Academic Press, New York.

Hawkins, J.E. Jt, (1973): Summary. In: Vascular Disorders and Hearing Defects, pp. 149 166. Editor: A.J. de Lorenzo. University Park Press, Baltimore.

Hawkins, J.E. Jr. (1976): Drug ototoxicity. In: Handbook of Sensory Physiology, Vol. V/3, pp. 707-748. Editors: W.D. Keidel and W.D. Neff, Springer-Verlag, Berlin.

Hilding, D.A. and Ginzberg, R.D. (1977): Pigmentation of the stria vascularis. Acta Otolaryngol. 84, 24-37.

Kimura, R.S. (1962): Experimental blockage of the endolymphatic duct and sac and its effect on the inner ear of the guinea pig. Ann. Otol. 76, 664-687.

Lawrence, M. (1983): Regeneration of Reissner's membrane. Acta Otolaryngol. 95, 480-485.

Longnecker, D.E (1980): The microcirculation. In: The Circulation in Anaesthesia, Applied Physiology and Pharmacology, pp. 167-178. Editor: C. Prys-Roberts. Blackwell Scicntific Publications, Oxford. 
Longnecker, D.E., Miller, F.N. and Harris, P.D. (1974): Small artery and vein response to ketamine $\mathrm{HCl}$ in the bat wing. Anesthesia Analgesia 53, 64.

Lyttkins, L., Larsson, B., Stahle, J. and Englesson, S. (1979): Accumulation of substances with melanin affinity to the internal ear. Adv. Oto-Rhino-Laryngol. 25, 17-25.

Majno, G.. Shea, S.M. and Levanthal, M. (1969): Endothelial contractions induced by histamine-type mediators. J. Cell Biol. 42, 647-672.

Mellander. S. and Johansson, B. (1968): Control of resistance, exchange and capacitance functions in the peripheral circulation. Pharmacol. Rev. 20, 117.

Rodriguez-Eschandia, E.L. and Burgos, M.H. (1965): The fine structure of the stria vascularis in the guinea pig. Z. Zellforsch. 67, 600

Santi, P.A. and Duvall, A.J. (1979): Morphological alteration of the stria vascularis after administration of the diuretic bumetanide. Acta Otolaryngol. 88, 1-12.

Santi, P.A. and Lakhani, B. (1983): A stereological analysis of the stria vascularis following bumetanide administration. Abstract. Sixth Midwinter Research Meeting, Association for Research in Otolaryngology, 1983, p. 42.

Santi, P.A., Lakhani, B. and Bingham, C. (1983): The volume density of cells and capillaries of the normal stria vascularis. Hearing Res. 11, 7-23
Smith, C.A. (1951): Capillary areas of the cochlea in the guinea pig. Laryngoscope 61, 10731985.

Smith, C.A. (1973): Vascular patterns of the membranous labyrinth. In: Vascular Disorders and Hearing Defects, pp. 1-21. Editor: A.J. de Lorenzo. University Park Press, Baltimore.

Smith, D.I., Lawrence, M. and Hawkins, J.E. Jr. (1985): Effects of noise and quinine on the vessels of the stria vascularis. Am. J. Otol. (in press).

Spoendlin, H. (1967): Vascular stria and spiral prominence. In: Submicroscopic Structure of the Inner Ear, pp. 131-147. Editor: S. Iurato, Pergamon Press, Oxford.

Tasaki, I. and Spyropoulos, C.S. (1959): Stria vascularis as source of endocochlear potential. J. Neurophysiol. 22 , 149-155.

Vertes, D. and Axelsson, A. (1979): Methodological aspects of some inner ear vascular techniques. Acta Otolaryngol. 88, $328-334$.

Vosteen, K. (1960): The histochemistry of the enzymes of oxygen metabolism in the inner ear. Laryngoscope 70 , $351-362$

Zar, J.H. (1980): Biostatistical Analysis, pp. 151-162. Prentice-Hall, New York.

Zweifach, B.W. (1971): E.M. Landis Award Acceptance Speech. Microvasc. Res. 3, 345-353. 